\title{
Rat vocalization to shock with and without a CS
}

Pietro badia, STEVE SUTER AND PAUL LeWIS

BOWLING GREEN STATE UNIVERSITY

Rat vocalization to electric shock was studied using a classical conditioning procedure for one group and presenting only the UCS to a second group. Ss given CS-UCS pairings vocalized significantly less to the UCS than Ss given only the UCS. Results were discussed and predicted from an information-preparation framework. Unexpected gradual UCR curves were obtained but no vocalization to the CS occurred.

One of the most striking and reliable behavioral observations of animals subjected to painful stimulation is the occurrence of repeated distress vocalizations during stimulation. This is especially common in the rat. The occurrence of such reliable responses provide the investigator with opportunities to identify the controlling variables of behavior and to discover generalized laws of conditioning using either operant or classical conditioning techniques.

The research reported here uses a classical conditioning procedure and deals with rat vocalizations to electric shock (UCS) but with emphasis on the unconditional response (UCR) instead of the typical emphasis on the conditioned response (CR). More specifically we are concerned with the number of vocalizations to electric shock when preceded by a warning stimulus (CS) in comparison with vocalizations when no CS is used. While vocalization to the UCS under these different conditions was our primary concern, we were, of course, also interested in the acquisition of vocalizations to the CS.

The theoretical frame of reference leading to our emphasis on the UCR instead of the CR is that of Perkins (1955). These theoretical statements diverge from traditional learning theory in that they state a CS paired with an aversive UCS does not necessarily acquire aversive properties. For Perkins, emphasis is on the informational value of the CS which allows the $S$ to prepare for the receipt of stimulation either aversive or attractive. This preparation based on information provided by the CS is said to minimize the painfulness of aversive stimuli or maximize the attractiveness of appetitive stimuli. Recent evidence supporting the information-preparation notions in both aversive and appetitive situations has been published (Lockhard, 1963; Perkins, Lewis, \& Seyman, 1963; Prokasy, 1965).

This study employs two groups and uses number of vocalizations to the UCS as the dependent variable. Group I Ss were given 36 paired CS-UCS trials while Group II Ss were given 36 trials with the UCS alone. Assuming the number of vocalizations to the UCS to be an indicator of its aversiveness, it was predicted Group I would vocalize less frequently to the shock than Group II, since the former group could presumably prepare for the receipt of shock and thus minimize its aversiveness.

\section{Method}

The Ss were 22 naive hooded rats, one-half males, and one-half females, 90 to 121 days of age at the beginning of training. There were $10 \mathrm{Ss}$ in the UCS group and $12 \mathrm{Ss}$ in the CS-UCS group. The uneven $\mathrm{N}$ occurred as a result of correcting an initial malefemale imbalance. However, one $S$ was discarded from the UCS group for failure to vocalize resulting in an $\mathrm{N}$ of 21 .

The apparatus was a clear Plexiglas compartment 9 in. long, 6 in. wide, and 7-1/2 in. deep with a grid floor of stainless steel rods. A 6 watt bulb located on the side panel served as a CS. The UCS was scrambled shock of .28 milliamps emitted by a Lehigh Valley Constant Current source. This low milliamp reading was used since pilot studies indicated all Ss vocalized at this level without ceiling effects. It should be noted that this level of shock is only slightly above the squeal threshold reported by Littman, Stevens \& Whittier (1964). Group I was given 36 CS-UCS pairings with an intertrial interval of $1 \mathrm{~min}$. using a delayed conditioning technique. The CS came on $3 \mathrm{sec}$. before the $2 \mathrm{sec}$. UCS and both terminated together. Group II was given 36 UCS presentations with all other conditions the same as the first group. A microphone was centered in the roof of the apparatus and connected to a tape recorder. Vocalizations were recorded at $7-1 / 2 \mathrm{in.} / \mathrm{sec}$. and played back and counted manually at $3-3 / 4 \mathrm{in.} / \mathrm{sec}$. Results

As seen in Fig. 1 the vocalization curves between the

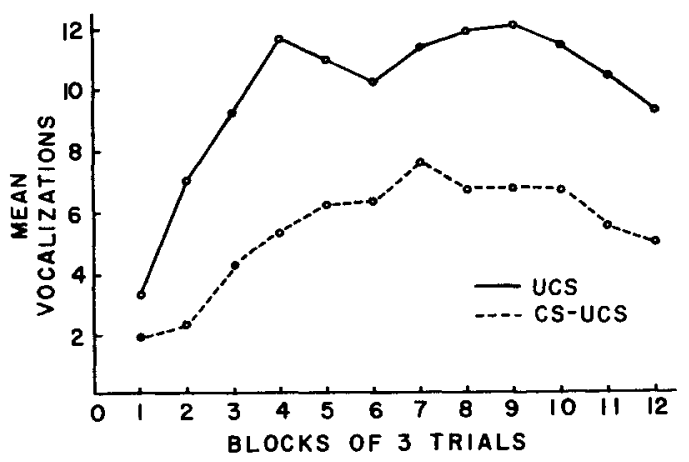

Fig. 1. Mean vocalization to the UCS in blocks of 3 trials for Ss given CS-UCS pairings or UCS alone. 
groups separate early and remain apart over the 36 training trials. While the initial differences during the first trial blocks are small, the curves begin to diverge early in training and appear to asymptote at the same rate. The predicted fewer number of vocalizations for the CS-UCS group in contrast to the group receiving the UCS alone was confirmed. However, quite unexpected was the appearance of gradual UCR acquisition curves for both groups.

Also unexpected was the total lack of vocalization to the CS for Group I. Perhaps more trials or training sessions are necessary to elicit vocalizations to the CS. No differences in vocalization occurred between males and females.

Statistical analyses of the reliability of the above observations were performed using a mixed design with the experimental conditions of Groups I and II as the between variable and blocks of six trials (Trials 1-24) as the within variable. The analysis for differences in vocalizations between the groups was significant, $F=$ 4.85 , df $=1 / 19, \mathrm{p}<.05$. Thus confirming the predicted observation that the CS-UCS group vocalized less to shock than the UCS group. While the trial blocks analysis also attained a rigorous significance level, $F=42.00$, $\mathrm{df}=3 / 57, \mathrm{p}<.005$, the interaction between groups and trial blocks did not, $F=1.97, \mathrm{df}=3 / 57$. These latter findings support the unexpected observation that UCR acquisition curves existed and that they did not interact with the experimental conditions.

\section{Discussion}

Assuming that frequency of vocalization reflects the aversiveness of the UCS, the finding that Ss vocalize less when given a warning signal (CS) preceding the UCS tends to support the information-preparation theoretical statements of Perkins (1955). Presumably responses made to the CS, considered preparatory in nature, minimize the aversiveness of the UCS and reduce the number of distress vocalizations. While taped recordings during the CS period did not indicate vocalizations to the $\mathrm{CS}$, observations of behavior during this period indicated the Ss were inactive but tended to assume certain postural responses. Following Lockard's suggestions (1963) there are several ways in which shock aversiveness can be reduced: shock avoidance, current reduction, reduction of arcing, decreased current density. Since a constant current scrambled shock source was used in the present study, shock avoidance and current reduction are unlikely candidates. However, and in agreement with Lockard, certain systematic postural responses, such as firmly gripping the grid bars with all four paws following CS onset, could have reduced current density by increasing contact area. Also, the arcing, characteristic of high voltage constant current sources, may have been minimized by the adoption of these postural responses. It should be

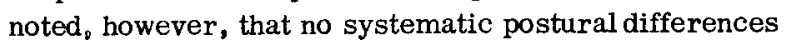
were observed during the UCS period between the two groups.

A final point not yet discussed is the gradual growth of the UCR curves for both groups. We are uncertain and puzzled as to their interpretation but two possibilities can be considered: increases in arousal or drive witr training, and superstitious operant vocalization.

First, drive or arousal could have increased with successive shocks. It seems unlikely, however, that the attainment of asymptotic drive levels would require 24 training trials. Further, motivational variables are usually defined in terms of their independence from practice and are characterized by rapid rather than gradual build up. For example, in other situations such as conditioned fear, asymptotic levels have been attained in fewer trials (Goldstein, 1960).

Second, the possibility of superstitious operant vocalization should be considered. Since vocalization occurred only during the short two-second shock period a superstitious contingency between vocalization and shock termination could have been acquired. The gradually increasing vocalization curves tend to support this latter speculation.

\section{References}

Goldstein, M. L. Acquired drive strength as a joint function of shock intensity and number of acquisition trials. J. exp. Psychol., 1960, 60, 349-358.

Littman, R. A., Stevens, D. A., \& Whittier, J. L. Previous shock experience and response threshold to shock. Canad. J. Psychol., $1964,18,93-100$.

Lockard, J. A. Choice of a warning signal or no warning signal in an unavoidable shock situation. J. comp. physiol. Psychol., $1963,56,526-530$.

Perkins, C. C., Jr. The stimulus conditions which follow learned responses. Psychol. Rev., 1955, 62, 341-348.

Perkins, C. C., Jr. Lewis, D. J., \& Seyman, R. Preference for signal-shock vs shock signal. Psychol. Rep., 1963, 13, 735-738.

Prokasy, W. F., Jr. The acquisition of observing responses in the absence of differential external reinforcement. J. comp. physiol. Psychol., 1956, 49, 131-134.

\section{Note}

1. This research was supported in part by USPHS Grant MH $08960-$ $01 \mathrm{~A} 1$ to the senior author. 